OPEN ACCESS

Edited by:

Simona Pisanti,

Università degli Studi di Salerno, Italy

Reviewed by:

Nikhil Tyagi,

University of South Alabama,

United States

Adolfo Rivero-Muller,

Turku Centre for Biotechnology,

Finland

*Correspondence:

Hsi-Hsien Lin

hhlin@mail.cgu.edu.tw

Specialty section:

This article was submitted to

Molecular and Cellular Oncology,

a section of the journal

Frontiers in Oncology

Received: 20 November 2017

Accepted: 19 July 2018

Published: 08 August 2018

Citation:

Huang K-Y and Lin H-H (2018) The Activation and Signaling Mechanisms of GPR56/ADGRG1 in Melanoma Cell.

Front. Oncol. 8:304

doi: 10.3389/fonc.2018.00304

\section{The Activation and Signaling Mechanisms of GPR56/ADGRG1 in Melanoma Cell}

\author{
Kuan-Yeh Huang ${ }^{1}$ and Hsi-Hsien Lin ${ }^{1,2,3 *}$ \\ ${ }^{1}$ Department of Microbiology and Immunology, College of Medicine, Chang Gung University, Taoyuan, Taiwan, ${ }^{2}$ Department \\ of Anatomic Pathology, Chang Gung Memorial Hospital-Linkou, Taoyuan, Taiwan, ${ }^{3}$ Chang Gung Immunology Consortium, \\ Chang Gung Memorial Hospital-Linkou, Taoyuan, Taiwan
}

Adhesion G protein-coupled receptors (aGPCRs) constitute the second largest GPCR subfamily. GPR56/ADGRG1 is a member of the ADGRG subgroup of aGPCRs. Although GPR56 is best known for its pivotal role in the cerebral cortical development, it is also important for tumor progression. Numerous studies have revealed that GPR56 is expressed in various cancer types with a role in cancer cell adhesion, migration and metastasis. In a recent study, we found that the immobilized GPR56-specific CG4 antibody enhanced IL-6 production and migration ability of melanoma cells. In this review, we will summarize the current understanding of GPR56 function and discuss the activation and signaling mechanisms of GPR56 in melanoma cells.

Keywords: GPR56, melanoma, tumor progression, signaling pathways, adhesion GPCR

\section{ADHESION GPCRS}

G protein-coupled receptor (GPCR) superfamily is the largest receptor family in nature. Because of their signature seven-transmembrane (7TM) domain, GPCRs are also called the 7TM receptors (1). Based on their phylogenetic relationship GPCRs are further classified into five subfamilies: Glutamate, Rhodopsin, Adhesion, Frizzled, and Secretin (GRAFS) (1). Most of the adhesion GPCRs (aGPCRs) were found initially through the genomic approaches and were orphan receptors due to the lack of interacting ligands. A total of 33 aGPCR members were identified in human and were subdivided into eight groups based on the genetic and structural criteria $(2,3)$. In recent years, a number of aGPCRs have been implicated in various physiological functions, genetic disorders as well as tumorigenesis (3).

Structurally distinct from the canonical GPCRs, aGPCRs contain an unusually large extracellular domain (ECD) composed of a diverse range of cell adhesive protein motifs normally arranged in tandem repeats at the N-terminal half (4-6). This is followed by a signature auto-proteolytic protein fold of $\sim 300$ residues, called the GPCR auto-proteolysis inducing (GAIN) domain, which is immediately upstream of the 7TM domain $(7,8)$. A highly conserved GPCR proteolysis site (GPS) is embedded in the GAIN domain proximal to the first TM region (9). During aGPCR synthesis and maturation, auto-proteolytic processing at GPS takes place in the endoplasmic reticulum or in the Gogi complex (9). This novel post-translational proteolytic reaction dissects the aGPCR into two subunits, a long extracellular N-terminal fragment (NTF, $\alpha$-subunit) and a 7TM C-terminal fragment (CTF, $\beta$-subunit). Interestingly, the cleaved NTF and CTF remain on the cell surface as a dual-component receptor complex via non-covalent interaction (9). 
It is now well-accepted that a major activation mechanism of aGPCRs involves the binding of the aGPCR-NTF with its ligand(s) followed by activation and intracellular signaling of the corresponding CTF (10-12). Indeed, the so called tethered agonism whereby a newly exposed $\mathrm{N}$-terminal agonist peptide of the CTF binds and activates the own CTF following the separation of the NTF from CTF has been shown for several aGPCRs $(13,14)$. In general, it is believed that the interaction of ligands/binding partners with the NTF in combination with mechanical disturbance lead to the detachment of the NTF-CTF complex and the subsequent manifestation of the Stachel agonist peptide for the activation of the own CTF. Nevertheless, the Stachel peptide-independent and GPS proteolysis-independent activation mechanisms have also been demonstrated for certain aGPCRs recently $(15,16)$. The activity and function of a given aGPCR hence is governed by multiple factors including the specific binding partners, receptor auto-proteolysis as well as micro-environmental changes.

\section{GPR56/ADGRG1}

GPR56/ADGRG1, first reported by Liu et al. and Zendman et al. $(17,18)$, belongs to the ADGRG subfamily of aGPCRs (3). Mapped on the short arm of human chromosome 16 (16q13) with a total of 13 exons, GPR56 (also named TM7XN1) was differentially expressed in a panel of melanoma cell lines of which the highly metastatic ones were found to display a markedly reduced GPR56 expression. Expressional analysis of transcript message indicated that GPR56 was widely distributed in various tissues including brain, heart, testis and thyroid gland $(17,18)$. In addition to the GAIN domain, an additional structurally-defined domain was identified recently. The Pentraxin/Laminin/neurexin/sex-hormone-binding-globulin-

Like (PLL) domain is located upstream of the GAIN domain at the most N-terminal region of the receptor (19). Herein, we will first review the biological and cellular functions of GPR56, followed by the identification of its binding partners/ligands, and finally the activation and signaling mechanisms of GPR56 in melanoma cells.

\section{BIOLOGICAL AND CELLULAR FUNCTIONS OF GPR56}

It is now known that GPR56 participates in a myriad of physiological processes. The best example is the link of GPR56 dysfunction to human developmental disorders (20-23). Indeed, GPR56 is critical for the frontal cortex development as lossof-function GPR56 mutations causally lead to a rare but severe brain cortical malformation called bilateral frontoparietal polymicrogyria (BFPP) (20). The different types of BFPPcausing GPR56 mutations are widespread and include deletional, splicing, frameshift, and missense point mutations (20). Most interestingly at least 12 distinct missense point mutations were identified in BFPP patients, most of them located at the extracellular region of GPR56 (20, 24, 25). These diseaseassociated missense mutations were especially instrumental in revealing multiple distinct BFPP-causing mechanisms mediated by GPR56 (25-28). In mice, Gpr56 gene deficiency resulted in neuronal malformations in the cerebral cortex essentially recapitulating the BFPP phenotype (29). This study established firmly the role of GPR56 in regulating the integrity of pial basement membrane (BM) during cortical development. More recently, it was further shown that regional cerebral cortical patterning was tightly regulated by the GPR56 expression levels controlled in part by a specific cis-regulatory non-exonic element and generation of multiple alternative splicing forms (30).

In addition to the cortical development, GPR56 is also involved in oligodendrocyte development and CNS myelination $(23,31)$. GPR56 expression was highest in the oligodendrocyte precursor cells (OPC), but declined gradually during oligodendrocyte maturation and was very low, if not absent, in mature myelinating oligodendrocytes $(23,32)$. Importantly, disruption of the GPR56 gene reduced the proliferation ability of OPC leading to a defect of CNS myelination (23). It was found that GPR56 regulated the proliferation of OPC via the $\mathrm{G} \alpha 12 / 13$ and RhoA signaling pathways (31). Hence, Gpr56 deficiency prompts the premature cell cycle exit of OPC, leading to less mature oligodendrocytes $(23,31)$. In a more recent study, GPR56 was shown to be also involved in myelin development and maintenance in the peripheral nervous system by interacting with plectin (33).

Next to the CNS development, GPR56 also plays a role in other developmental processes such as muscle cell differentiation, male fertility and immune regulation. As such, upregulated GPR56 expression is detected during early myoblast differentiation. Importantly, GPR56 deletion causes primary myoblast cell abnormality in vitro, but muscle development in vivo was not affected (34). Moreover, a role in regulating muscle cell hypertrophy under resistance exercise conditions was noted recently for GPR56, which likely signaled via the $G \alpha_{12 / 13}$ and mTOR pathways (35). In the reproductive system, GPR56 was involved in the testis development and male fertility as loss of GPR56 lead to reduced fertility in male mice due to partial disruption of seminiferous tubules. GPR56 was thought to be essential for the establishment of asymmetric testis cord remodeling during male gonad development (21). In the immune system, GPR56 is restrictedly expressed in cytotoxic lymphocytes including NK cells $(36,37)$. Subsequent investigation by us showed that GPR56 functions as an inhibitory receptor on human NK cells through cis-interaction with the tetraspanin CD81 (38).

Apart from the aforementioned biological and cellular functions, increasing evidences also implicate a critical role of GPR56 in tumor cell progression (39-41). Apart from the early description of its differential expression in melanoma cells, GPR56 expression was also detected in other cancer cell types including esophageal squamous cell (42), breast, nonsmall cell lung, ovarian, colon, pancreatic carcinoma $(43,44)$, and glioblastoma/astrocytoma (45). These studies indicated strongly a role of GPR56 in tumor cell adhesion, migration and survival/apoptosis (39-45). In addition, GPR56 is also strongly expressed in a subgroup of acute myeloid leukemia (AML) containing high levels of the ecotropic viral integration 
site-1 (EVI1) transcription factor (40). Interestingly, GPR56 gene is identified as a transcriptional target of EVI1 and increased GPR56 expression in EVI1-high AML promotes cell adhesion and anti-apoptotic activities (40). Furthermore, GPR56 is identified recently as a marker of human leukemia stem cells (LSCs), which give rise to AML. The GPR56 expression level in LSCs is positively correlated with high-risk AML diseases and poor clinical outcome. Engrafting GPR56-overexpressing AML cells into mice accelerates myeloid leukemogenesis and decreases the survival rate $(40,46,47)$. Importantly, functional blockage of GPR56 by specific antibody efficiently attenuated AML cell engraftment suggesting GPR56 as a potential anti-leukemic target $(40,46,47)$. In addition, GPR56 was found to have an inhibitory role in the mesenchymal differentiation and radioresistance of glioblastoma by negatively regulating the nuclear factor kappa $\mathrm{B}$ (NF-кB) signaling pathway (48). Despite these advances, the role of GPR56 in tumor progression was investigated in depth mostly in melanoma cells, which we will discuss in the later section.

\section{LIGANDS/BINDING PARTNERS OF GPR56}

As a canonical aGPCR, it is not surprising that several cellular ligands/binding partners have been identified for GPR56 in past years (Figure 1). The identification and functions of these cellular ligands/binding partners of GPR56 are discussed below.

\section{COLLAGEN III}

Collagen III (col-III), encoded by the Col3al gene, is expressed mostly in skin and blood vessel and is involved in the normal development of these tissues (49). While searching for a GPR56specific ligand in brain, Piao's group found that GPR56-NTF interacted specifically with col-III which is highly expressed in the meninges, pial BM, and blood vessels during mouse embryonic development (50). The col-III binding site of GPR56 was located at a.a. 27-160 and several BFPP-associated point mutations including R38Q, R38W, Y88C, and C91S all lost the col-III binding ability (28). Collagen III-GPR56 interaction activates $\mathrm{G} \alpha_{12 / 13}$ and RhoA pathways and inhibits the migration of neural cells in vitro (50-52). Functional analysis of the Col3a1 ${ }^{-/-}$mice identified very similar phenotypes of brain malformation seen in the GPR56 null animals, including over migration of neurons and a cobblestone-like appearance (50). Therefore, it is concluded that col-III is a major GPR56-ligand in the developing brain and acts to regulate the normal lamination process of the cerebral cortex. A later study showed that col-III binding to GPR56 facilitates the separation and release of GPR56-NTF from the GPR56-CTF, which translocate from the non-lipid raft to the lipid raft membrane regions and induces RhoA activation (52).

\section{TANSGLUTAMINASE 2 (TG2)}

In clinical observation, the expression level of GPR56 is correlated inversely to the metastatic ability of melanoma cells, suggesting an inhibitory role in melanoma metastasis $(12,18,39)$. Xu's group first demonstrated the presence of a putative GPR56ligand in the extracellular matrix (ECM) of lung, stomach, and tumor tissues using the GPR56-NTF as a probe. They subsequently identified transglutaminase 2 (TG2) as the ECMligand of GPR56 in melanoma tissues (39). It was found that the C-terminal region of TG2 interacts directly with the serine threonine proline-rich (STP) segment of the GPR56NTF (39, 53). Importantly, it was shown that the binding of TG2 to GPR56 triggered the endocytosis process so that TG2 was internalized and degraded in the lysosome (54). This led to reduced deposition of fibronectin in the ECM and attenuated repertoire of focal adhesion kinase. These results indicated an antagonistic GPR56-TG2 relationship in regulating ECM deposition and cell-ECM adhesion during melanoma cell progression $(39,54)$. It is likely that TG2 is the dominant tissue environmental determinant regulating the metastatic potential of GPR56-expressing melanoma cells (55).

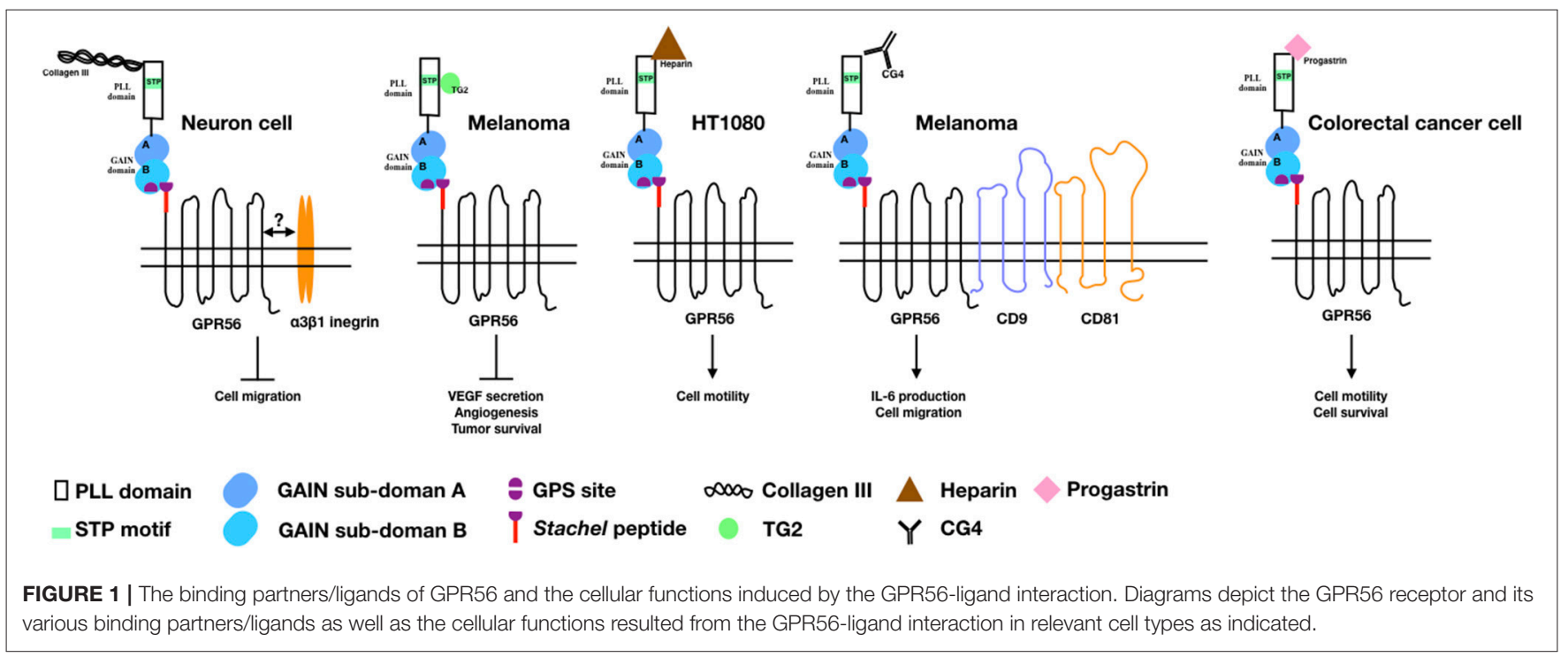




\section{HEPARIN}

Besides the two protein ligands described above, heparin was verified by us recently as a novel glycosaminoglycan (GAG) binding partner of GPR56 (56). Interestingly, several aGPCRs including EMR2/ADGRE1, CD97/ADGRE5, and GPR124/ADGRA2 had also been shown to interact with distinct GAGs, suggesting a ubiquitous aGPCR-GAG partnership (57-59). The putative heparin-binding sites of GPR56 are mapped to two regions rich in basic residues at a.a. 26-35 and a.a. 190-200, which overlap partially with the col-III and TG2-binding sites. Most interestingly, both col-III and TG2 are also heparin-binding proteins, but only col-III is able to modulate the GPR56-heparin interaction (56). Unexpectedly, heparin binding attenuated GPR56-NTF shedding leading to enhanced cell adhesion and motility (56). This finding highlights the functional complexity of the interaction of GPR56 with its diverse endogenous ligands.

\section{CIS-INTERACTING MOLECULES}

While the role of cellular ligands/binding partners that interact with GPR56 in-trans has been delineated, a couple of putative cisinteracting molecules of GPR56 have also been identified. Little at al. showed that GPR56 formed a complex with the tetraspanin CD81 and $\mathrm{G} \alpha_{\mathrm{q} / 11}$ from which at least two distinct activation mechanisms can be induced. It was concluded that CD81 probably acted as a GPCR-scaffolding protein that promotes and stabilizes the GPR56 signaling complex (60). Indeed, a role of CD81 in regulating GPR56 receptor function in human NK cell was verified by us recently (38). In addition, a functional role of a putative GPR56 accessory molecule, the $\alpha_{3} \beta_{1}$ integrin, in brain development has also been demonstrated by Jeong et al. (51). Previous studies have shown that GPR56-deficient granule cells reduced its adhesion to laminin, a ligand of the $\alpha_{3} \beta_{1}$ integrin that is also involved in the pial BM integrity (51). Interestingly, the cortical phenotype was exacerbated in the GPR $56 / \alpha_{3}$-integrin double knock out animals. The results indicate strongly a synergistic collaboration between GPR56 and $\alpha 3 \beta 1$ integrin in regulating the cortical development (51). Intriguingly, most tetraspanin molecules such as CD9, CD81 and CD151, are known to associate with the integrin proteins on membrane (61-63). Hence, these data suggest the presence of a possible GPR56-tetraspanin-integrin super-complex that awaits further investigation.

\section{PROGASTRIN}

Progastrin, a growth factor and potent pro-angiogenic factor, was identified recently as a soluble ligand of GPR56 (41). Progestin has been known to enhance the proliferation of colonic epithelial cells and the progression of colorectal cancer in mice (6466). Jin et al. showed that overexpression of human progastrin in mice stimulated GPR56 expression in colonic mucosa cells. Furthermore, progastrin was able to bind to cell surface GPR56 and the proliferation of GPR56-expressing colorectal cancer cells was increased in the presence of progastrin (41). Likewise, progastrin treatment enhanced the growth and survival of colonic organoids derived from the wild-type but not GPR56null mice (41). Progastrin-dependent colonic proliferation in vivo was inhibited by the loss of GPR56. Finally, GPR56 deficiency also abrogated progastrin-dependent colonic crypt fission and colorectal carcinogenesis in a mouse model of colorectal cancer (41). Hence, the progastrin-GPR56 axis is thought as an important promoter of colorectal tumorigenesis in vivo.

\section{GPR56-SPECIFIC ABS, MONOBODIES, AND SMALL-MOLECULE LIGAND}

Over the years, the development of aGPCR-specific reagents such as antibodies (Abs) has facilitated the functional delineation of the receptors. Indeed, several GPR56-specific Abs have been produced to help dissect its activation mechanism and cellular functions. Iguchi et al. first detected strong GPR56 expression in neural progenitor cells (NPCs) using polyclonal Abs against the GPR56-NTF. GPR56 overexpression in NPCs was shown to negatively regulated cell migration. Importantly, they subsequently identified an agonistic activity of the GPR56specific polyclonal Abs which were able to functionally inhibit NPC migration in a $\mathrm{G}_{12} / 13^{-}$and Rho-dependent manner (67). In recent years, we have generated five mouse monoclonal antibodies (mAbs) against human GPR56 (37). One of the mAbs, CG4, was found to activate GPR56 in melanoma cells when immobilized on the plate (12). Specifically, it was shown that interaction with the immobilized CG4 ligated and activated GPR56 on melanoma cells to enhance IL-6 secretion in a CD9/CD81-dependent manner via the $\mathrm{G} \alpha_{12 / 13^{-}}$and Rhosignaling pathways (12). The mechanistic mechanism of CG4induced GPR56 activation in melanoma cell involves the shedding of GPR56-NTF and will be discussed in detail in the later section.

Ohta et al. similarly generated several GPR56 mAbs, some of them were able to inhibit human U87-MG glioma cell migration via the $\mathrm{G}_{\mathrm{q}^{-}}$and Rho-signaling pathways. Interestingly, the agonistic mAbs were found to enhance NTF-CTF interaction as revealed by co-immunoprecipitation analysis (68). More recently, Salzman et al. succeed in selecting engineered monobodies with high specificity and affinity to the GPR56-NTF. These monobodies not only help stabilize and determine the crystal structure of the GPR56-NTF and the identification of the novel PLL domain, but also reveal a Stachel-independent NTFmediated activation mechanism of GPR56 $(16,19)$. The effect of the GPR56-specific monobodies on cellular functions however remains unexplored. Finally, Stoveken et al. identified a smallmolecule ligands of GPR56, dihyromunduletone (DHM) and 3$\alpha$-acetoxydihydrodeoxygedunin (3- $\alpha$-DOG) which is antagonist and agonist respectively, from a library of compounds using a reporter-based screening strategy $(69,70)$. DHM and 3- $\alpha$-DOG modulated GPR56 activation induced by the tethered as well as the exogenous synthetic Stachel peptides. Interestingly, DHM and 3- $\alpha$-DOG also regulated the activation of GPR114/ADGRG5 apparently due to the highly similar Stachel peptide sequence shared with GPR56. Anyhow, DHM and 3- $\alpha$-DOG represent 
small-molecule aGPCR ligands capable of modulating aGPCR activity.

\section{ALTERNATIVE SPLICE VARIANTS OF GPR56}

While most studies focused on the ligand-receptor interaction or the expression level of GPR56 during tumor progression, the composition of GPR56 protein variants might also influence the tumorigenesis. A total of five GPR56 receptor variants have been identified, including the full-length wild-type and four alternative splice variants (71). Variants 1 and 2 both missed eight amino acids present at the first intracellular loop, with the variant 2 contains an extra five residues at the cleavage site of the signal peptide. WT, variants 1 and 2 promote the downstream factors expression not only including the SRE response elements, but also COX2, iNOS, and VEGF which contributed to tumor growth (71). Both variants 3 and 4 exhibit a large deletion compared to the WT. Variant 3 contains a 170-residue deletion (38-208) which truncated the PLL domain (19). Translation of variant 4 starts with ATG at the exon $3(19,71)$. These results further confirmed a critical role of the extracellular PLL domain in regulating GPR56 function (19). Hence, it is of importance to consider the splice variants of GPR56 when examining its role in tumorigenesis.

\section{THE ACTIVATION AND SIGNALING MECHANISMS OF GPR56/ADGRG1 IN MELANOMA CELL}

The GPR56-mediated signaling pathways have been investigated by several groups in diverse cell types. These include the GPR56/CD81/G $\alpha_{\mathrm{q} / 11}$ complex described in HEK293 and U937 cells by Little et al. (60) and the GPR56 polyclonal Ab-induced $\mathrm{G} \alpha_{12 / 13}$ and RhoA pathway in NPCs by Iguchi et al. (67). Subsequent studies on oligodendrocytes and other cell types also highlighted the involvement of the $\mathrm{G} \alpha_{12 / 13}$ and RhoA pathways $(12,31,50,72)$. Herein, we will focus on the activation and signaling mechanism of GPR56 in melanoma cells.

Consistent with the earlier report of reduced expression of GPR56 transcripts in highly metastatic melanoma cell lines, $\mathrm{Xu}$ et al. showed that GPR56 was significantly downregulated in highly metastatic cells in an in vivo metastatic model of human melanoma cells (39). The inhibitory role of GPR56 in melanoma tumor growth and metastasis was further confirmed using specific RNAi-mediated gene silence. Indeed, TG2 was identified in this study as a potential ECM ligand that contributed to the metastatic suppressive function of GPR56. This is likely due to the antagonistic effect of TG2-GPR56 interaction on the deposition of ECM and ECM-cell adhesion as mentioned above (54). Furthermore, GPR56 was shown to suppress the angiogenesis and growth of melanoma by inhibiting the production of vascular endothelial growth factor (VEGF) via

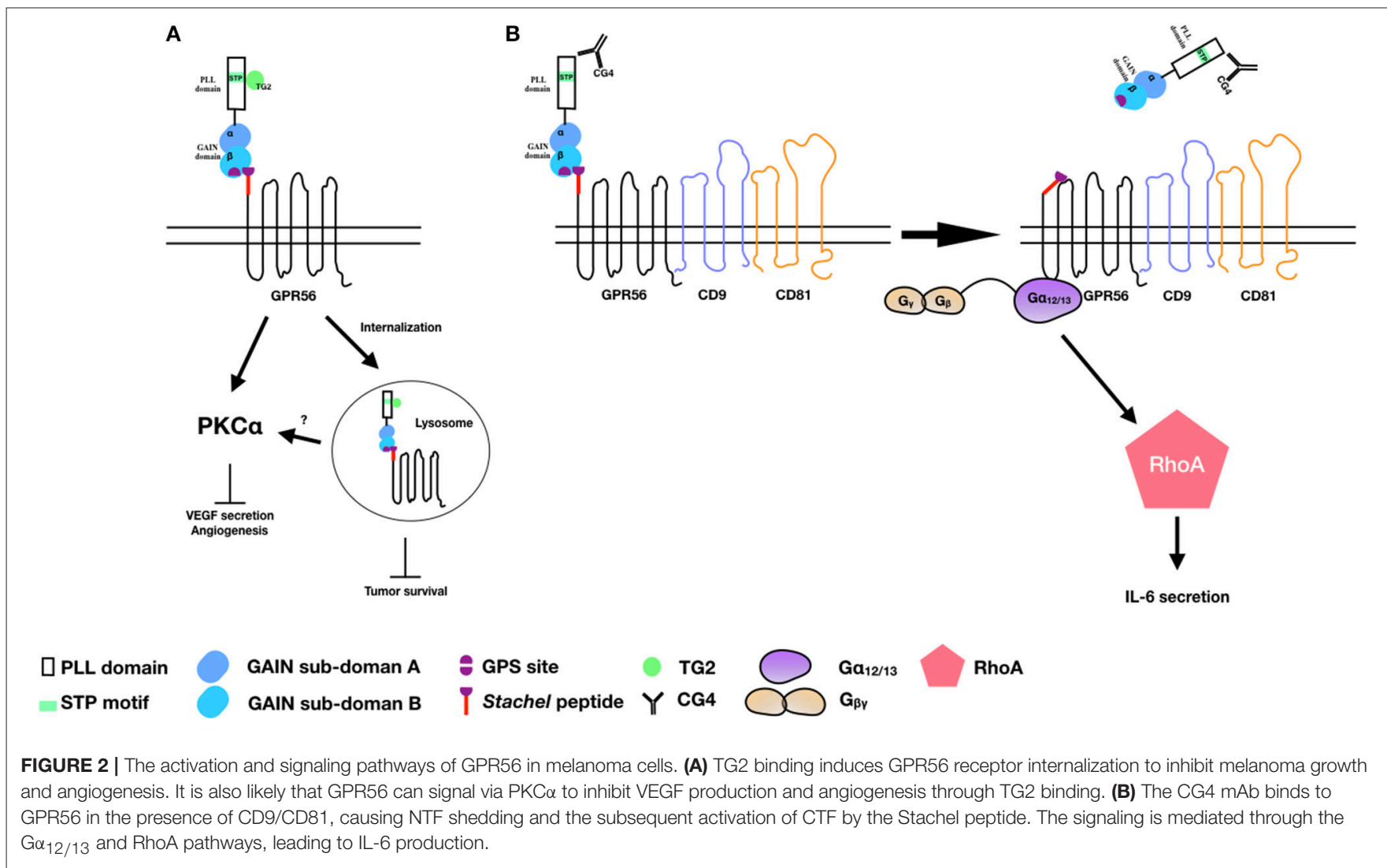


the PKC $\alpha$ signaling pathway in a STP domain-dependent manner (53). Because the STP domain is the TG2-binding region, these results implied that by binding to the STP domain, TG2 induced GPR56-mediated inhibition of VEGF secretion and angiogenesis via the inactivation of PKC $\alpha$ (Figure 2A) (53).

By contrast, we showed that through the binding and ligation of immobilized CG4 mAb, GPR56 receptor cross-linking in melanoma cells promoted the shedding of GPR56-NTF and subsequent activation and signaling of GPR56-CTF via the $\mathrm{G} \alpha_{12 / 13}$ and RhoA pathways, resulting in IL-6 production. Interestingly, the CG4-induced NTF shedding required the presence of CD9/CD81 as CD9/CD81 knockdown significantly reduced IL-6 secretion. It was further shown that the dissociation of GPR56-NTF unmasked the Stachel peptide of GPR56-CTF, which was responsible for activating CTF and the signaling transduction. Hence, our results are in line with the tethered agonist model of aGPCRs of which the receptor activation requires the release of the NTF of a fully GPS-cleaved receptor in a Stachel peptide-dependent manner (Figure 2B) (12).

While these studies provide important insights into the role of GPR56 in tumorigenesis, especially in melanoma progression, our understanding of GPR56 function in tumor biology is just at its infancy. For example, conflicting outcomes were observed in the involvement of GPR56 in the development of endogenous tumors (73). More recently, alternative GPS proteolysis-independent and Stachel-independent mechanisms of GPR56 activation have been uncovered. It is very likely that GPR56 is involved in different stages of tumor cell progression by multiple activation pathways.

\section{REFERENCES}

1. Fredriksson R, Lagerstrom MC, Lundin LG, Schioth HB. The G-proteincoupled receptors in the human genome form five main families. Phylogenetic analysis, paralogon groups, and fingerprints. Mol Pharmacol. (2003) 63:125672. doi: $10.1124 / \mathrm{mol} .63 .6 .1256$

2. Bjarnadottir TK, Fredriksson R, Hoglund PJ, Gloriam DE, Lagerstrom MC, Schioth HB. The human and mouse repertoire of the adhesion family of G-protein-coupled receptors. Genomics (2004) 84:23-33. doi: 10.1016/j.ygeno.2003.12.004

3. Hamann J, Aust G, Arac D, Engel FB, Formstone C, Fredriksson R, et al. International Union of Basic and Clinical Pharmacology. XCIV. Adhesion G protein-coupled receptors. Pharmacol Rev. (2015) 67:338-67. doi: 10.1124/pr.114.009647

4. Baud V, Chissoe SL, Viegas-Pequignot E, Diriong S, N'Guyen VC, Roe BA, et al. EMR1, an unusual member in the family of hormone receptors with seven transmembrane segments. Genomics (1995) 26:334-44.

5. Krasnoperov VG, Bittner MA, Beavis R, Kuang Y, Salnikow KV, Chepurny OG, et al. alpha-Latrotoxin stimulates exocytosis by the interaction with a neuronal G-protein-coupled receptor. Neuron (1997) 18:925-37.

6. Kwakkenbos MJ, Kop EN, Stacey M, Matmati M, Gordon S, Lin HH, et al. The EGF-TM7 family: a postgenomic view. Immunogenetics (2004) 55:655-66. doi: 10.1007/s00251-003-0625-2

7. Arac D, Boucard AA, Bolliger MF, Nguyen J, Soltis SM, Sudhof TC, et al. A novel evolutionarily conserved domain of cell-adhesion GPCRs mediates autoproteolysis. EMBO J. (2012) 31:1364-78. doi: 10.1038/emboj. 2012.264

8. Promel S, Langenhan T, Arac D. Matching structure with function: the GAIN domain of adhesion-GPCR and PKD1-like proteins. Trends Pharmacol Sci. (2013) 34:470-8. doi: 10.1016/j.tips.2013.06.002

\section{CONCLUSIONS AND DISCUSSIONS}

GPR56/ADGRG1 not only involves in the normal tissue development but also in tumorigenesis. Previous studies have identified numerous cellular binding partners/ligands and developed specific Abs/monobodies of GPR56. Meanwhile, distinct GPR56-mediated signaling pathways are being revealed. These unique reagents provide excellent research tools for the functional delineation of GPR56 and identify GPR56 as an important player in melanoma development. Functional studies with these GPR56-specific reagents including mAbs, monobodies, and small-molecule compounds have demonstrated effective modulation of its signaling activities (16, 46, 70). It is hoped that better understanding of GPR56-mediated activation and signaling mechanisms as well as the development of GPR56-sepcific reagents in the future may help target GPR56 as a potential cancer therapeutic molecule, especially in melanoma.

\section{AUTHOR CONTRIBUTIONS}

$\mathrm{K}-\mathrm{YH}$ and $\mathrm{H}-\mathrm{HL}$ drafting and writing of the manuscript.

\section{ACKNOWLEDGMENTS}

We would like to acknowledge the grant support from the Ministry of Science and Technology, Taiwan (MOST104-2320-B-182-035-MY3) and the Chang Gung Memorial Hospital (CORPD1F0061 and BMRP860).

9. Lin HH, Chang GW, Davies JQ, Stacey M, Harris J, Gordon S. Autocatalytic cleavage of the EMR2 receptor occurs at a conserved G proteincoupled receptor proteolytic site motif. J Biol Chem. (2004) 279:31823-32. doi: 10.1074/jbc.M402974200

10. Hu QX, Dong JH, Du HB, Zhang DL, Ren HZ, Ma ML, et al. Constitutive Galphai coupling activity of very large G protein-coupled receptor 1 (VLGR1) and its regulation by PDZD7 protein. J Biol Chem. (2014) 289:24215-25. doi: 10.1074/jbc.M114.5498168

11. Peeters MC, Fokkelman M, Boogaard B, Egerod KL, van de Water B, IJzerman AP, et al. The adhesion G protein-coupled receptor G2 (ADGRG2/GPR64) constitutively activates SRE and NFkappaB and is involved in cell adhesion and migration. Cell Signal. (2015) 27:2579-88. doi: 10.1016/j.cellsig.2015.08.015

12. Chiang NY, Peng YM, Juang HH, Chen TC, Pan HL, Chang GW, et al. GPR56/ADGRG1 activation promotes melanoma cell migration via NTF dissociation and CTF-mediated Galpha12/13/RhoA signaling. J Invest Dermatol. (2017) 137:727-36. doi: 10.1016/j.jid.2016.10.031

13. Liebscher I, Schon J, Petersen SC, Fischer L, Auerbach N, Demberg LM, et al. A tethered agonist within the ectodomain activates the adhesion $\mathrm{G}$ protein-coupled receptors GPR126 and GPR133. Cell Rep. (2014) 9:2018-26. doi: 10.1016/j.celrep.2014.11.0361

14. Stoveken HM, Hajduczok AG, Xu L, Tall GG. Adhesion G protein-coupled receptors are activated by exposure of a cryptic tethered agonist. Proc Natl Acad Sci USA. (2015) 112:6194-9. doi: 10.1073/pnas.14217851120

15. Kishore A, Purcell RH, Nassiri-Toosi Z, Hall RA. Stalk-dependent and Stalk-independent signaling by the adhesion $G$ protein-coupled receptors GPR56 (ADGRG1) and BAI1 (ADGRB1). J Biol Chem. (2016) 291:3385-94. doi: 10.1074/jbc.M115.6893490

16. Salzman GS, Zhang S, Gupta A, Koide A, Koide S, Arac D. Stachelindependent modulation of GPR56/ADGRG1 signaling by synthetic ligands 
directed to its extracellular region. Proc Natl Acad Sci USA. (2017) 114:10095100. doi: 10.1073/pnas.17088101147

17. Liu M, Parker RM, Darby K, Eyre HJ, Copeland NG, Crawford J, et al. GPR56, a novel secretin-like human G-protein-coupled receptor gene. Genomics (1999) 55:296-305. doi: 10.1006/geno.1998.5644

18. Zendman AJ, Cornelissen IM, Weidle UH, Ruiter DJ, van Muijen GN. TM7XN1, a novel human EGF-TM7-like cDNA, detected with mRNA differential display using human melanoma cell lines with different metastatic potential. FEBS Lett. (1999) 446:292-8.

19. Salzman GS, Ackerman SD, Ding C, Koide A, Leon K, Luo R, et al. Structural basis for regulation of GPR56/ADGRG1 by its alternatively spliced extracellular domains. Neuron (2016) 91:1292-304. doi: 10.1016/j.neuron.2016.08.0221

20. Piao X, Hill RS, Bodell A, Chang BS, Basel-Vanagaite L, Straussberg R, et al. $\mathrm{G}$ protein-coupled receptor-dependent development of human frontal cortex. Science (2004) 303:2033-6. doi: 10.1126/science.1092780

21. Chen G, Yang L, Begum S, Xu L. GPR56 is essential for testis development and male fertility in mice. Dev Dyn. (2010) 239:3358-67. doi: 10.1002/dvdy.224680

22. Singer K, Luo R, Jeong SJ, Piao X. GPR56 and the developing cerebral cortex: cells, matrix, and neuronal migration. Mol Neurobiol. (2013) 47:186-96. doi: 10.1007/s12035-012-8343-03

23. Giera S, Deng Y, Luo R, Ackerman SD, Mogha A, Monk KR, et al. The adhesion G protein-coupled receptor GPR56 is a cell-autonomous regulator of oligodendrocyte development. Nat Commun. (2015) 6:6121. doi: 10.1038/ncomms71215

24. Piao X, Chang BS, Bodell A, Woods K, Benzeev B, Topcu M, et al. Genotypephenotype analysis of human frontoparietal polymicrogyria syndromes. Ann Neurol. (2005) 58:680-7. doi: 10.1002/ana.20616

25. Ke N, Ma H, Diedrich G, Chionis J, Liu G, Yu DH, et al. Biochemical characterization of genetic mutations of GPR56 in patients with bilateral frontoparietal polymicrogyria (BFPP). Biochem Biophys Res Commun. (2008) 366:314-20. doi: 10.1016/j.bbrc.2007.11.071

26. Jin Z, Tietjen I, Bu L, Liu-Yesucevitz L, Gaur SK, Walsh CA, et al. Disease-associated mutations affect GPR56 protein trafficking and cell surface expression. Hum Mol Genet. (2007) 16:1972-85. doi: 10.1093/hmg/ddm144

27. Chiang NY, Hsiao CC, Huang YS, Chen HY, Hsieh IJ, Chang GW, et al. Disease-associated GPR56 mutations cause bilateral frontoparietal polymicrogyria via multiple mechanisms. J Biol Chem. (2011) 286:14215-25. doi: $10.1074 /$ jbc.M110.1838308

28. Luo R, Jin Z, Deng Y, Strokes N, Piao X. Disease-associated mutations prevent GPR56-collagen III interaction. PLoS ONE (2012) 7:e29818. doi: 10.1371/journal.pone.00298182

29. Li S, Jin Z, Koirala S, Bu L, Xu L, Hynes RO, et al. GPR56 regulates pial basement membrane integrity and cortical lamination. J Neurosci. (2008) 28:5817-26. doi: 10.1523/JNEUROSCI.0853-08.20083

30. Bae BI, Tietjen I, Atabay KD, Evrony GD, Johnson MB, Asare E, et al. Evolutionarily dynamic alternative splicing of GPR56 regulates regional cerebral cortical patterning. Science (2014) 343:764-8. doi: $10.1126 /$ science. 12443928

31. Ackerman SD, Garcia C, Piao X, Gutmann DH, Monk KR. The adhesion GPCR Gpr56 regulates oligodendrocyte development via interactions with Galpha12/13 and RhoA. Nat Commun. (2015) 6:6122. doi: $10.1038 /$ ncomms71222

32. Mehta P, Piao X. Adhesion G-protein coupled receptors and extracellular matrix proteins: roles in myelination and glial cell development. Dev Dyn. (2017) 246:275-84. doi: 10.1002/dvdy.244731

33. Ackerman SD, Luo R, Poitelon Y, Mogha A, Harty BL, D'Rozario M, et al. GPR56/ADGRG1 regulates development and maintenance of peripheral myelin. J Exp Med. (2018) 215:941-61. doi: 10.1084/jem.201617142

34. Wu MP, Doyle JR, Barry B, Beauvais A, Rozkalne A, Piao X, et al. G-protein coupled receptor 56 promotes myoblast fusion through serum response factor- and nuclear factor of activated T-cell-mediated signalling but is not essential for muscle development in vivo. FEBS J. (2013) 280:6097-113. doi: $10.1111 /$ febs. 125292

35. White JP, Wrann CD, Rao RR, Nair SK, Jedrychowski MP, You JS, et al. G protein-coupled receptor 56 regulates mechanical overloadinduced muscle hypertrophy. Proc Natl Acad Sci USA. (2014) 111:15756-61. doi: 10.1073/pnas.14178981118
36. Della Chiesa M, Falco M, Parolini S, Bellora F, Petretto A, Romeo E, et al. GPR56 as a novel marker identifying the CD56dull CD16+ NK cell subset both in blood stream and in inflamed peripheral tissues. Int Immunol. (2010) 22:91-100. doi: 10.1093/intimm/dxp116

37. Peng YM, van de Garde MD, Cheng KF, Baars PA, Remmerswaal EB, van Lier RA, et al. Specific expression of GPR56 by human cytotoxic lymphocytes. $J$ Leukoc Biol. (2011) 90:735-40. doi: 10.1189/jlb.0211092

38. Chang GW, Hsiao CC, Peng YM, Vieira Braga FA, Kragten NA, Remmerswaal $\mathrm{EB}$, et al. The adhesion G protein-coupled receptor GPR56/ADGRG1 is an inhibitory receptor on human NK cells. Cell Rep. (2016) 15:1757-70. doi: 10.1016/j.celrep.2016.04.053

39. Xu L, Begum S, Hearn JD, Hynes RO. GPR56, an atypical G proteincoupled receptor, binds tissue transglutaminase, TG2, and inhibits melanoma tumor growth and metastasis. Proc Natl Acad Sci USA. (2006) 103:9023-8. doi: 10.1073/pnas.06026811034

40. Saito Y, Kaneda K, Suekane A, Ichihara E, Nakahata S, Yamakawa $\mathrm{N}$, et al. Maintenance of the hematopoietic stem cell pool in bone marrow niches by EVI1-regulated GPR56. Leukemia (2013) 27:1637-49. doi: $10.1038 /$ leu.2013.75

41. Jin G, Sakitani K, Wang H, Jin Y, Dubeykovskiy A, Worthley DL, et al. The Gprotein coupled receptor 56 , expressed in colonic stem and cancer cells, binds progastrin to promote proliferation and carcinogenesis. Oncotarget (2017) 8:40606-19. doi: 10.18632/oncotarget.165060

42. Sud N, Sharma R, Ray R, Chattopadhyay TK, Ralhan R. Differential expression of G-protein coupled receptor 56 in human esophageal squamous cell carcinoma. Cancer Lett. (2006) 233:265-70. doi: 10.1016/j.canlet.2005. 03.018

43. Ke N, Sundaram R, Liu G, Chionis J, Fan W, Rogers C, et al. Orphan G protein-coupled receptor GPR56 plays a role in cell transformation and tumorigenesis involving the cell adhesion pathway. Mol Cancer Ther. (2007) 6:1840-50. doi: 10.1158/1535-7163.MCT-07-0066

44. Liu Z, Huang Z, Yang W, Li Z, Xing S, Li H, et al. Expression of orphan GPR56 correlates with tumor progression in human epithelial ovarian cancer. Neoplasma (2017) 64:32-9. doi: 10.4149/neo_2017_104

45. Shashidhar S, Lorente G, Nagavarapu U, Nelson A, Kuo J, Cummins J, et al. GPR56 is a GPCR that is overexpressed in gliomas and functions in tumor cell adhesion. Oncogene (2005) 24:1673-82. doi: 10.1038/sj.onc.1208395

46. Daria D, Kirsten N, Muranyi A, Mulaw M, Ihme S, Kechter A, et al. GPR56 contributes to the development of acute myeloid leukemia in mice. Leukemia (2016) 30:1734-41. doi: 10.1038/leu.2016.76

47. Pabst C, Bergeron A, Lavallee VP, Yeh J, Gendron P, Norddahl GL, et al. GPR56 identifies primary human acute myeloid leukemia cells with high repopulating potential in vivo. Blood (2016) 127:2018-27. doi: 10.1182/blood-2015-11-683649

48. Moreno M, Pedrosa L, Pare L, Pineda E, Bejarano L, Martinez J, et al. GPR56/ADGRG1 inhibits mesenchymal differentiation and radioresistance in glioblastoma. Cell Rep. (2017) 21:2183-97. doi: 10.1016/j.celrep.2017.10.083

49. Kim JK, Xu Y, Xu X, Keene DR, Gurusiddappa S, Liang X, et al. A novel binding site in collagen type III for integrins alphalbetal and alpha2betal. J Biol Chem. (2005) 280:32512-20. doi: 10.1074/jbc.M502431200

50. Luo R, Jeong SJ, Jin Z, Strokes N, Li S, Piao X. G protein-coupled receptor 56 and collagen III, a receptor-ligand pair, regulates cortical development and lamination. Proc Natl Acad Sci USA. (2011) 108:12925-30. doi: $10.1073 /$ pnas. 11048211087

51. Jeong SJ, Luo R, Singer K, Giera S, Kreidberg J, Kiyozumi D, et al. GPR56 functions together with $\alpha 3 \beta 1$ integrin in regulating cerebral cortical development. PLoS ONE (2013) 8:e68781. doi: 10.1371/journal.pone.00687811

52. Luo R, Jeong SJ, Yang A, Wen M, Saslowsky DE, Lencer WI, et al. Mechanism for adhesion G protein-coupled receptor GPR56-mediated RhoA activation induced by collagen III stimulation. PLOS ONE (2014) 9:e100043. doi: 10.1371/journal.pone.01000439

53. Yang L, Chen G, Mohanty S, Scott G, Fazal F, Rahman A, et al. GPR56 Regulates VEGF production and angiogenesis during melanoma progression. Cancer Res. (2011) 71:5558-68. doi: 10.1158/0008-5472.CAN-10-45438

54. Yang L, Friedland S, Corson N, Xu L. GPR56 inhibits melanoma growth by internalizing and degrading its ligand TG2. Cancer Res. (2014) 74:1022-31. doi: 10.1158/0008-5472.CAN-13-12681 
55. Xu L, Hynes RO. GPR56 and TG2: possible roles in suppression of tumor growth by the microenvironment. Cell Cycle (2007) 6:160-5. doi: $10.4161 /$ cc.6.2.3760

56. Chiang NY, Chang GW, Huang YS, Peng YM, Hsiao CC, Kuo ML, et al. Heparin interacts with the adhesion GPCR GPR56, reduces receptor shedding, and promotes cell adhesion and motility. J Cell Sci. (2016) 129:215669. doi: $10.1242 /$ jcs. 174458

57. Stacey M, Chang GW, Davies JQ, Kwakkenbos MJ, Sanderson RD, Hamann J, et al. The epidermal growth factor-like domains of the human EMR2 receptor mediate cell attachment through chondroitin sulfate glycosaminoglycans. Blood (2003) 102:2916-24. doi: 10.1182/blood-2002-11-3540

58. Wang T, Ward Y, Tian L, Lake R, Guedez L, Stetler-Stevenson WG, et al. CD97, an adhesion receptor on inflammatory cells, stimulates angiogenesis through binding integrin counterreceptors on endothelial cells. Blood (2005) 105:2836-44. doi: 10.1182/blood-2004-07-2878

59. Vallon M, Essler M. Proteolytically processed soluble tumor endothelial marker (TEM) 5 mediates endothelial cell survival during angiogenesis by linking integrin alpha(v)beta3 to glycosaminoglycans. J Biol Chem. (2006) 281:34179-88. doi: 10.1074/jbc.M605291200

60. Little KD, Hemler ME, Stipp CS. Dynamic regulation of a GPCRtetraspanin-G protein complex on intact cells: central role of CD81 in facilitating GPR56-Galpha q/11 association. Mol Biol Cell. (2004) 15:2375-87. doi: 10.1091/mbc.E03-12-08867

61. Spring FA, Griffiths RE, Mankelow TJ, Agnew C, Parsons SF, Chasis JA, et al. Tetraspanins CD81 and CD82 facilitate $\alpha 4 \beta 1$-mediated adhesion of human erythroblasts to vascular cell adhesion molecule-1. PLoS ONE (2013) 8:e62654. doi: 10.1371/journal.pone.00626542

62. Reyes R, Monjas A, Yanez-Mo M, Cardenes B, Morlino G, Gilsanz A, et al. Different states of integrin LFA-1 aggregation are controlled through its association with tetraspanin CD9. Biochim Biophys Acta (2015) 1853:2464-80. doi: 10.1016/j.bbamcr.2015.05.018

63. Yu J, Lee CY, Changou CA, Cedano-Prieto DM, Takada YK, Takada Y. The CD9, CD81, and CD151 EC2 domains bind to the classical RGD-binding site of integrin alphavbeta3. Biochem J. (2017) 474:589-96. doi: 10.1042/BCJ20160998

64. Wang TC, Koh TJ, Varro A, Cahill RJ, Dangler CA, Fox JG, et al. Processing and proliferative effects of human progastrin in transgenic mice. J Clin Invest. (1996) 98:1918-29. doi: 10.1172/JCI118993

65. Jin G, Ramanathan V, Quante M, Baik GH, Yang X, Wang SS, et al. Inactivating cholecystokinin-2 receptor inhibits progastrin-dependent colonic crypt fission, proliferation, and colorectal cancer in mice. J Clin Invest. (2009) 119:2691-701. doi: 10.1172/JCI389184
66. Jin G, Westphalen CB, Hayakawa Y, Worthley DL, Asfaha S, Yang X, et al. Progastrin stimulates colonic cell proliferation via CCK2R- and beta-arrestindependent suppression of BMP2. Gastroenterology (2013) 145:820-30 e10. doi: 10.1053/j.gastro.2013.07.0346

67. Iguchi T, Sakata K, Yoshizaki K, Tago K, Mizuno N, Itoh H. Orphan G protein-coupled receptor GPR56 regulates neural progenitor cell migration via a G alpha 12/13 and Rho pathway. J Biol Chem. (2008) 283:14469-78. doi: 10.1074/jbc.M708919200

68. Ohta S, Sakaguchi S, Kobayashi Y, Mizuno N, Tago K, Itoh H. Agonistic antibodies reveal the function of GPR56 in human glioma U87-MG cells. Biol Pharm Bull. (2015) 38:594-600. doi: 10.1248/bpb.b1400752

69. Stoveken HM, Bahr LL, Anders MW, Wojtovich AP, Smrcka AV, Tall GG. Dihydromunduletone Is a small-molecule selective adhesion G protein-coupled receptor antagonist. Mol Pharmacol. (2016) 90:214-24. doi: $10.1124 / \mathrm{mol} .116 .1048281$

70. Stoveken HM, Larsen SD, Smrcka AV, Tall GG. Gedunin- and Khivorinderivatives are small molecule partial agonists for adhesion $\mathrm{G}$ protein coupled receptors GPR56/ADGRG1 and GPR114/ADGRG5. Mol Pharmacol. (2018) 93:477-88. doi: 10.1124/mol.117.111476

71. Kim JE, Han JM, Park CR, Shin KJ, Ahn C, Seong JY, et al. Splicing variants of the orphan G-protein-coupled receptor GPR56 regulate the activity of transcription factors associated with tumorigenesis. J Cancer Res Clin Oncol. (2010) 136:47-53. doi: 10.1007/s00432-009-0635-Z

72. Paavola KJ, Stephenson JR, Ritter SL, Alter SP, Hall RA. The N terminus of the adhesion G protein-coupled receptor GPR56 controls receptor signaling activity. J Biol Chem. (2011) 286:28914-21. doi: 10.1074/jbc.M111.24 79736

73. Xu L, Begum S, Barry M, Crowley D, Yang L, Bronson RT, et al. GPR56 plays varying roles in endogenous cancer progression. Clin Exp Metastasis (2010) 27:241-9. doi: 10.1007/s10585-010-9322-30

Conflict of Interest Statement: The authors declare that the research was conducted in the absence of any commercial or financial relationships that could be construed as a potential conflict of interest.

Copyright (C) 2018 Huang and Lin. This is an open-access article distributed under the terms of the Creative Commons Attribution License (CC BY). The use, distribution or reproduction in other forums is permitted, provided the original author(s) and the copyright owner(s) are credited and that the original publication in this journal is cited, in accordance with accepted academic practice. No use, distribution or reproduction is permitted which does not comply with these terms. 